\title{
Circumbinary Planets Orbiting the Rapidly Pulsating Subdwarf B-type binary NY Vir
}

\author{
Qian S.-B. ${ }^{1,2,3}$, Zhu L.-Y.1,2, Dai Z.-B. ${ }^{1,2}$, Fernández Lajús, E. ${ }^{4,5}$, Xiang F.-Y. ${ }^{6}$, and He, \\ J.-J.1,2
}

\begin{abstract}
We report here the tentative discovery of a Jovian planet in orbit around the rapidly pulsating subdwarf B-type (sdB-type) eclipsing binary NY Vir. By using new determined eclipse times together with those collected from the literature, we detect that the observed-calculated (O-C) curve of NY Vir shows a smallamplitude cyclic variation with a period of 7.9 years and a semiamplitude of $6.1 \mathrm{~s}$, while it undergoes a downward parabolic change (revealing a period decrease at a rate of $\dot{P}=-9.2 \times 10^{-12}$ ). The periodic variation was analyzed for the lighttravel time effect via the presence of a third body. The mass of the tertiary companion was determined to be $M_{3} \sin i^{\prime}=2.3( \pm 0.3) M_{\text {Jupiter }}$ when a total mass of $0.60 M_{\odot}$ for NY Vir is adopted. This suggests that it is most probably a giant circumbinary planet orbiting NY Vir at a distance of about 3.3 astronomical units (AU). Since the rate of period decrease can not be explained by true angular momentum loss caused by gravitational radiation or/and magnetic braking, the observed downward parabolic change in the O-C diagram may be only a part of a long-period (longer than 15 years) cyclic variation, which may reveal the presence of another Jovian planet $\left(\sim 2.5 M_{\text {Jupiter }}\right)$ in the system.
\end{abstract}

Subject headings: Stars: binaries : close - Stars: binaries : eclipsing - Stars: individuals (NY Vir) - Stars: subdwarfs - Stars: planetary system

\footnotetext{
${ }^{1}$ National Astronomical Observatories/Yunnan Observatory, Chinese Academy of Sciences (CAS), P.O. Box 110, 650011 Kunming, P.R. China (e-mail: qsb@ynao.ac.cn)

${ }^{2}$ Key laboratory of the structure and evolution celestial bodies, Chinese Academy of Sciences, P. O. Box 110, 650011 Kunming, P. R. China

${ }^{3}$ Graduate University of the Chinese Academy of Sciences, Yuquan Road 19\#, Sijingshang Block, 100049 Beijing, P. R. China

${ }^{4}$ Facultad de Ciencias Astronómicas y Geofísicas, Universidad Nacional de La Plata, 1900 La Plata, Buenos Aires, Argentina

${ }^{5}$ Instituto de Astrofisica de La Plata (CCT La plata - CONICET/UNLP), Argentina
} 


\section{Introduction}

HW Vir-like eclipsing binaries are a group of detached binary systems that consists a very hot subdwarf B-type (sdB) primary and a fully convective M-type secondary with periods shorter than 4 hours (e.g., Menzies \& Marang 1986; Kilkenny et al. 1998; Drechsel et al. 2001; Ostensen et al. 2007; Polubek et al. 2007; Wils et al. 2007; For et al. 2010; Ostensen et al. 2010). The hot sdB components in this group of systems are on the extreme horizontal branch (EHB) of the Hertzsprung-Russell diagram burning helium in their cores and and having very thin hydrogen envelopes (e.g., Heber 2009). Theoretical investigations have shown that they are formed through a common-envelope evolution (e.g., Han et al. 2003), and will evolve into normal cataclysmic variables (CV) (e.g., Shimansky et al. 2006). The discovery of circumbinary substellar objects orbiting these peculiar binaries has very important implications of several outstanding problems in astrophysics, e.g., the formation of sdB stars and the fates of low-mass companion systems. To date, three substellar companions were found in HW Vir and HS0705+6700 systems as circumbinary brown dwarfs and planets (Lee et al. 2009; Qian et al. 2009).

As for substellar objects in orbits around single sdB-type stars, Silvotti et al. (2007) discovered a giant planet with a mass of $3.2 M_{\text {Jupiter }}$ and an orbital separation of $1.7 \mathrm{AU}$, around the hot sdB star V391 Peg. Geier et al. (2009) later announced the detection of a close substellar companion to the hot sdB star HD 149382 with a short period of 2.391 days. However, Jacobs et al. (2011) found no evidence for the presence of the claimed substellar object by analyzing He lines. Recently, Geier et al. (2011) detected a brown dwarf companion to the hot subdwarf SDSS J083053.53+000843.4 with a very short period of 0.096 days and a mass of $0.045-0.067 M_{\odot}$. Here we report the tentative discovery of a Jovian planet with

a mass of $\sim 2.3 M_{\text {Jupiter }}$ around the HW Vir-like binary star NY Vir. The planet has the lowest mass among the substellar objects discovered before orbiting sdB stars. Moreover, evidence may show that there is another circumbinary planet companion in the system.

\section{New observations and changes of the $\mathrm{O}-\mathrm{C}$ diagram}

NY Vir (=PG 1336-018) was discovered as a sdB star by the Palomar-Green survey (Green et al. 1986) and was later found to be a HW Vir-like eclipsing binary by Kilkenny et al. (1998). It is a short-period detached system with a period between 2 and 3 hours. The sdB primary is also a rapid pulsator and the secondary is an $M_{5}$-type star. The detailed photometric and spectroscopic investigation was carried out by Kilkenny et al. (1998) who determined a mass ratio of 0.3 . They also found effective temperatures $T_{1}=33000 \mathrm{~K}$ and $T_{2}=3000 \mathrm{~K}$. A multisite (WET) campaign identified 28 pulsation frequencies in the $\mathrm{sdB}$ 


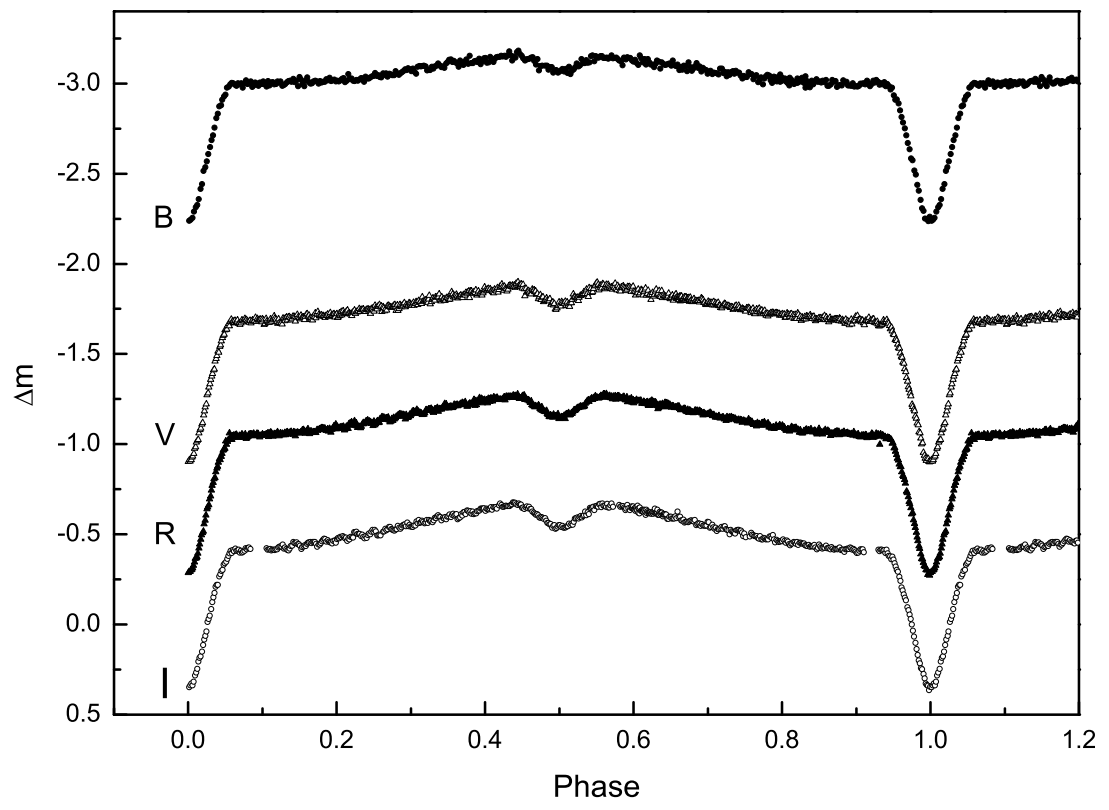

Fig. 1. - The light curves of NY Vir in B, V, R, and I bands observed in the time interval from May 28 to 31, 2011. Phases of the observations were calculated with the linear ephemeris: Min.I $=$ HJD 2455711.65991+0.101015999 $\times E$. 
star and found that the amplitudes of at least the strongest frequencies were varying on timescales of days (Kilkenny et al. 2003).

38 times of light minimum of NY Vir were published by using the telescopes in South African Astronomical Observatory (SAAO) (Kilkenny et al. 1998, 2000, 2011). Linear ephemerides,

$$
\text { Min.I }(H J D)=2450223.36142+0.1010174 \times E
$$

and

$$
\operatorname{Min} . I(H J D)=2450223.36134+0.101015999 \times E
$$

were determined by Kilkenny et al. (1998 and 2000), where E is the cycle number. A recent period investigation of NY Vir (Kilkenny et al. 2011) suggests that its period is decreasing at a rate of $\dot{P}=-11.2 \times 10^{-13} \mathrm{~d}$ per orbit. The mechanism that causes this decrease is unknown. To get more data for this binary star, it was observed with the 1.0-m telescope in Yunnan observatory on April 20, 2007. Then, after read the paper by Kilkenny et al. (2011), we monitored it again in the time interval from May 28 to 31, 2011 with the 2.15-m "Jorge Sahade" telescope in Argentina. During the observation a Versarray 1300B CCD camera was used. The complete light curves in B, V, R, and I bands were obtained and are displayed in Fig. 1. By using those photometric data, 9 eclipse times were determined. In order to obtain a high data precision, we applied a standard time system Barycentric Julian Dynamical Date (BJD), which are converted by the code of Stumpff (1980), to all eclipse timings and orbital period analysis. The derived eclipse times in BJD are listed in Table 1.

With all of available times of light minimum, the linear ephemeris published by Kilkenny et al. (1998 and 2000) was revised as,

$$
\operatorname{Min} . I(B J D)=2450223.362213(8)+0.1010159673(2) \times E,
$$

where BJD 2450223.362213 is the initial epoch, and 0.1010159673 is the revised period. The variance is derived as $1^{d} .7 \times 10^{-4}$. The $(O-C)_{1}$ values respect to the linear ephemeris were calculated. The corresponding $(O-C)_{1}$ diagram is plotted in Fig. 2. As shown in the figure, a downward parabolic fit (dashed line in Fig. 2) can not describe the general trend of the $(O-C)_{1}$ curve good. This indicates that, to describe the general $(O-C)_{1}$ trend satisfactorily, a combination of a downward parabolic change and a cyclic oscillation is required. Using the least squares method, we determined,

$$
\begin{aligned}
(O-C)_{1}= & -0.000073( \pm 0.00002)+2.7( \pm 0.2) \times 10^{-8} \times E \\
& -4.6( \pm 0.4) \times 10^{-13} \times E^{2} \\
& +0.000071( \pm 0.000017) \sin \left[0 .^{\circ} 0126( \pm 0.0007) \times E+312 .^{\circ} 1\left( \pm 20 .^{\circ} 1\right)\right],
\end{aligned}
$$




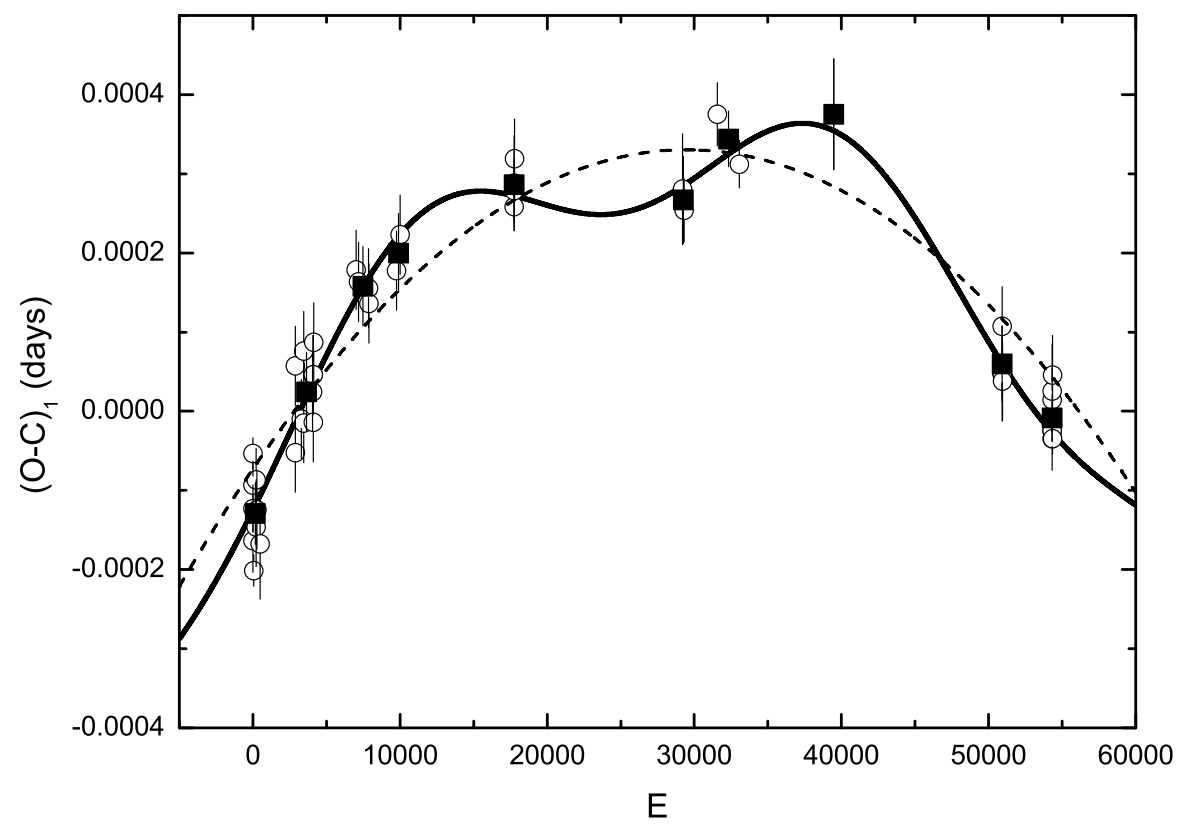

Fig. 2. - The O-C diagram of the sdB-type eclipsing binary NY Vir respect to Eq. (3). The solid line refers to the best fit of the $\mathrm{O}-\mathrm{C}$ curve by a combination of a periodic variation with a small amplitude of $6.1 \mathrm{~s}$ and a period of 7.9 years and a downward parabolic change (the dashed line). Open circles refer to the original data points, while solid squares to their averaged values. 
with a variance of $3^{d} .8 \times 10^{-5}$ and the $\chi^{2} \approx 1.2$. Using the F-test as discussed by Pringle (1975) to assess the significance of quadratic and sinusoidal terms in Eq. 4, the parameter $\lambda$ can be derived to be 180 , which indicates that it is significant well above $99.99 \%$ level. The rate of the orbital period decrease is close to that determined by Kilkenny et al. (2011). Considering that the influence of the pulsations on the minima time measurements is a plausible reason for the observed season scatters shown in Fig. 2 and 3, we average over the eclipse times within one year as a window of time. The averaged points are well obey our fitting curve in a long base line. Thus, we believed that the variation in O-C diagram should be real.

After the downward parabolic variation was removed, the $(O-C)_{2}$ curve is plotted in upper panel of Fig. 3 where a cyclic change is seen more clearly. The cyclic variation has an amplitude of 6.1 seconds and a period of 7.9 years. As pointed out by Kilkenny et al. (2011), eclipsing times for NY Vir are less accurate than for AA Dor because of the pulsating. However, errors introduced should be of the order of $0.00005 \mathrm{~d}$ or less (Kilkenny et al. 2001) and essentially random. The mean values of $(\mathrm{O}-\mathrm{C})_{2}$ suggest that the systematically cyclic variation in the $(\mathrm{O}-\mathrm{C})_{2}$ diagram is true. After all of the changes were removed, the residuals are shown in the lower panel of Fig. 3. It can be seen in the panel that the scatter of the residuals is in a random way indicating the scatter may be caused by the pulsating of the sdB star. Moreover, no systematic changes can be traced there, which suggests that Eq. (4) can give a good fit to the observations.

\section{Discussions and conclusions}

Investigations of several authors (e.g., Qian et al. 2008; Brinkworth et al. 2005) showed that the magnetic activity cycles of a fully convective component star (i. e., the Applegate mechanism) (Applegate 1992) can not explain the observed cyclic variations in the O-C diagrams of close binaries because of the problem of energy. Moreover, a recently statistical study by Liao and Qian (2010) suggests that light travel-time effect is the most probable mechanism to cause cyclic changes in the O-C diagrams of close binary stars. Therefore, we analyzed NY Vir for the light travel-time effect that arises from the gravitational influence of a tertiary companion. The possible presence of a companion object produces the relative distance changes of the eclipsing pair as it orbits the barycenter of the triple system.

By considering a typical mass of $M_{1}=0.46 M_{\odot}$ for sdB-type stars and using the mass ratio of $q=0.3$ determined by Kilkenny et al. (1998), the mass of the secondary is estimated as $M_{2}=0.14 M_{\odot}$. Then, the mass function and the mass of the possible tertiary companion 


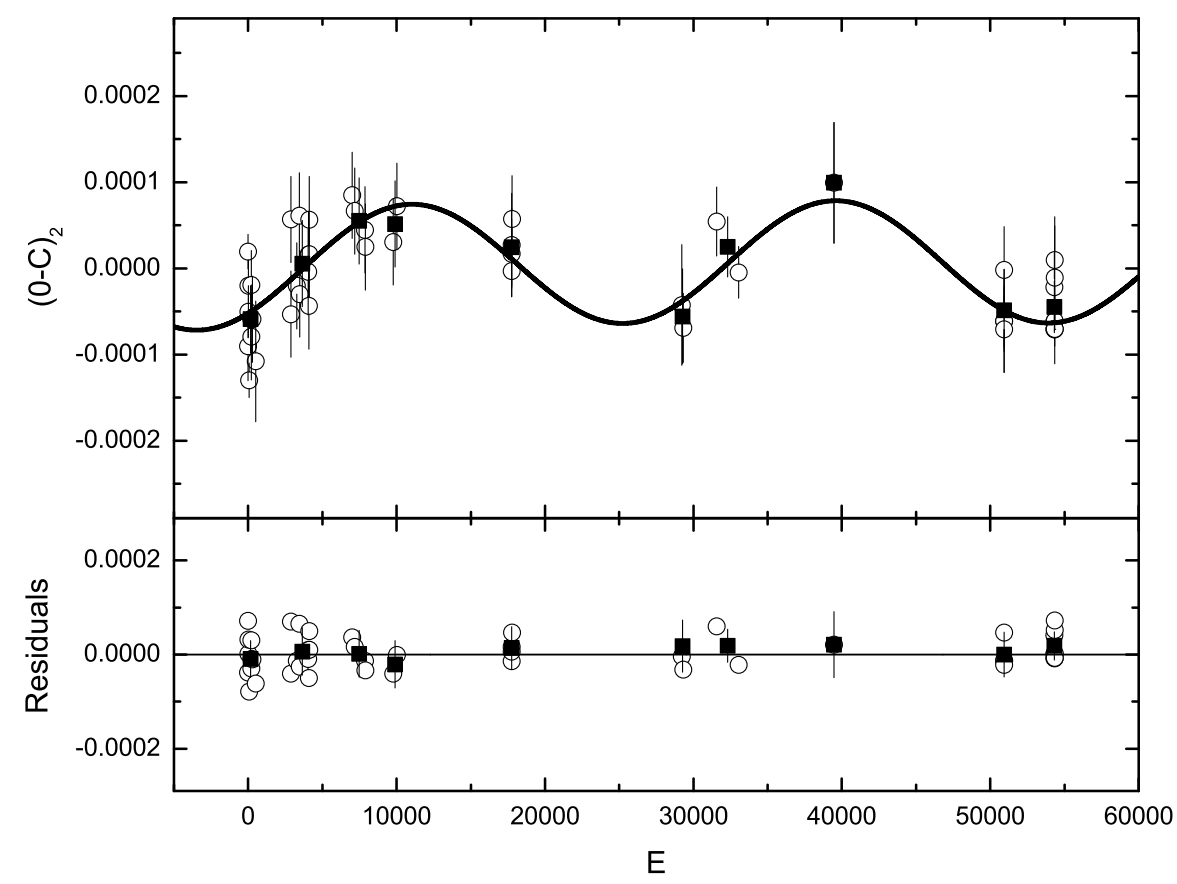

Fig. 3. - The cyclic variation of NY Vir (the light travel-time effect via the presence of the giant circumbinary planet) after the downward parabolic change is subtracted from the $(O-C)_{1}$ diagram. Symbols are the same as those in Fig. 2. The mean values follow the general $(O-C)_{2}$ trend, which suggests that the cyclic variation is real. 
were derived by using the following equation,

$$
f(m)=4 \pi^{2}\left(a_{12} \sin i^{\prime}\right)^{3} / G T^{2}=\left(M_{3} \sin i^{\prime}\right)^{3} /\left(M_{1}+M_{2}+M_{3}\right)^{2},
$$

where $M_{3}$ is the mass of the third body, $\mathrm{G}$ is the gravitational constant, and $\mathrm{T}$ is the period of the $(O-C)_{2}$ oscillation. $a_{12} \sin i^{\prime}$ can be determined by,

$$
a_{12} \sin i^{\prime}=A \times c
$$

where $\mathrm{A}$ is the semi-amplitude of the $(\mathrm{O}-\mathrm{C})_{2}$ oscillation and $c$ the speed of light. The results are: $f(m)=3.0( \pm 0.4) \times 10^{-8} M_{\odot}$ and $M_{3} \sin i^{\prime}=2.3( \pm 0.3) M_{\text {Jupiter }}$. When the orbital inclination of the third body is larger than $9.6^{\circ}$, the mass of the tertiary component

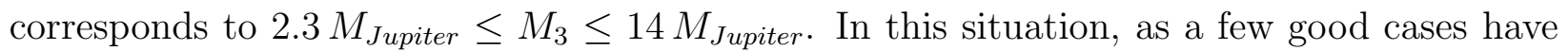
been made for circumbinary planets (e.g., Lee et al. 2009; Beuermann et al., 2010; Qian et al. 2010, 2011), it should be a planetary object. The orbital separation $d_{3}$ of the Jupiter-like planet candidate is about $3.3( \pm 0.8) \mathrm{AU}$.

The downward parabolic change in the O-C diagram (the dashed line in Fig. 2) implies a period decrease at a rate of $\dot{P}=-9.2 \times 10^{-12}$ (or 1 s in about 3,447 years). This corresponds to $\dot{P}=-3.36 \times 10^{-9}$ days/year, which is close to the value obtained in literature (Kilkenny et al. 2011). The orbital separation of the two component stars in NY Vir is estimated as $0.77 R_{\odot}$. By using the equation given by Kraft et al. (1961) and Faulkner (1971),

$$
\frac{\dot{P}_{G R}}{P}=-3 \frac{32 G^{3}}{5 c^{5}} \frac{M_{1} M_{2}\left(M_{1}+M_{2}\right)}{d^{4}},
$$

where $P$ is the orbital period and $d$ the distance between both components, the contribution of gravitational radiation (GR) to the period decrease can be computed as $\dot{P}_{G R}=-0.026 \times$ $10^{-9}$ days/year. This is about two orders smaller than the observed value indicating that it can not be caused by GR. This decrease can be explained by magnetic braking (MB) as in the case of HW Vir (Qian et al. 2008; Lee et al. 2009). However, it is more widely accepted that MB is stopped for fully convective stars (Rappaport et al. 1983; Spruit \& Ritter 1983).

Therefore, as in the case of HU Aqr, the more plausible reason for the observed longterm period decrease is that it is only a part of a long-period cyclic variation (longer than 15 years), revealing the possible presence of another planet in the planetary system. This is the same as that of HU Aqr where a planetary system is orbiting the eclipsing polar (Qian et al. 2011). We estimate that the mass of the second planet is about $2.5 M_{\text {Jupiter }}$. Some investigators (e.g., Horner et al. 2011) think that the serious problem for the presence of the fourth body is the dynamical stability of the circumbinary planetary system. However, the 3D dynamic-aware analysis of the stability of circumbinary orbits by Doolin et al. (2011) 
reveals that these orbits are surprisingly stable throughout binary mass fraction - eccentricity parameter space.

If the cyclic variations in the $\mathrm{O}-\mathrm{C}$ diagram are caused by the light-travel time effects via the presence of planetary objects, they should be strictly periodic. To check the existence of the planetary system, more eclipse times are needed in the future. Moreover, At the maximum point of the O-C curve, the central eclipsing binary NY Vir is at the farthest position of the orbit, while the circumbinary planet is closest to the observer. If the tertiary companion is coplanar to the central binary, the circumbinary planet should be in the light of the eclipsing binary and transit the binary component stars. Therefore, searching for the transits of the binary components by the circumbinary planets at the O-C maxima can ascertain the planetary system. More recently, a Saturn-like planet transits an M-type eclipsing binary was reported by Doyle et al. (2011), which provides the first direct evidence for the presence of a circumbinary planet.

As in the cases of other HW Vir-like binary stars, NY Vir was evolved through a common envelope (CE) after the more massive component star in the original system evolves into a red giant. The ejection of CE removed a large amount of the angular momentum, and the present EHB star with a low-mass stellar companion in a short-period binary was formed. The distance between the central EHB binary and the circumbinary planet is about 3.3 AU. By assuming the mass of the main-sequence (MS) progenitor of the EHB star is $M_{M S}=1.0 M_{\odot}$, the orbital separation of the planet around the MS progenitor is estimated as (e.g., Bear \& Soker 2011): $a_{0} \cong\left(M_{E H}+M_{2}\right) /\left(M_{M S}+M_{2}\right) \approx 1.74 \mathrm{AU}$ (by ignoring tidal interaction). When the primary star evolved to a red giant branch star before the system entered a $\mathrm{CE}$, it has a reached radius of $\sim 100 R_{\odot} \sim 0.5 \mathrm{AU}$. Therefore, the secondary should be at a distance of a little large, say $0.8 \mathrm{AU}$. Can a system of two stars $\left(M_{1}=1.0 M_{\odot}\right.$ and $\left.M_{2}=0.14 M_{\odot}\right)$ at an orbital separation of $0.8 \mathrm{AU}$ have a planet at $1.74 \mathrm{AU}$ in a stable orbit? The 3D numerical simulations by Doolin et al. (2011) indicates that the orbit is stable. A system with two M-type stars $\left(M_{1}=0.69 M_{\odot}\right.$ and $\left.M_{2}=0.20 M_{\odot}\right)$ at an orbital separation of $0.22 \mathrm{AU}$ was reported to have a Saturn-like planetary companion at a distance of $0.7 \mathrm{AU}$ (Doyle et al. 2011). If the detection of a planetary companion to NY Vir at orbital separation of 3.3 AU is true, it can give some constraints on the stellar evolution and the interaction between red giants and their planetary companions. Moreover, it is interesting to pointed out that the orbital separations between the central binaries and the inner substellar objects in the three systems, i.e., HW Vir (Lee et al. 2009), HS 0705+6700 (Qian et al. 2009), and NY Vir, are all in a small range of 3.3-3.6 AU. It is unclear whether these agreements are fortuitous or there are physical reasons.

This work is partly supported by Chinese Natural Science Foundation (No. 11133007, 
No.11003040, and No.10878012) and by the West Light Foundation of the Chinese Academy of Sciences. New CCD photometric observations of NY Vir were obtained with the 1.0-cm telescope in Yunnan observatory and with the 2.15-m "Jorge Sahade" telescope.

\section{REFERENCES}

Applegate, J. H., 1992, ApJ 385, 621

Bear, E. \& Soker, N., 2011, MNRAS 411, 1792

Beuermann, K., Hessman, F. V., Dreizler, S., et al., 2010, A\&A 521, L60

Brinkworth, C. S., Marsh, T. R., Dhillon, V. S., Knigge, C., 2006, MNRAS 365, 287

Doolin, S. \& Blundell, K. M., 2011, MNRAS.tmp.1777D

Doyle, L. R., Carter, J. A., Fabrycky, D. C., et al., 2011, Science 333, 1602.

Drechsel, H., Heber, U., Napiwotzki, R., Ostensen, R., Solheim, J.-E., Johannessen, F., Schuh, S. L., Deetjen, J., Zola, S., 2001, A\&A 379, 893

Faulkner, J., 1971, ApJ 170, 99

For, B.-Q., Green, E. M., Fontaine, G., Drechsel, H., Shaw,J. S., et al., 2010, ApJ 708, 253

Geier, S., Edelmann, H., Heber, U., Morales-Rueda, L., 2009, ApJ 702, L96

Geier, S., Schaffenroth, V., Drechsel, H., Heber, U., Kupfer, T., et al., 2011, ApJ 731, L22

Green, R. F., Schmidt, M., Liebert, J., 1986, ApJS 61, 305

Han, Z., Podsiadlowski, Ph., Maxted, P. F. L., Marsh, T. R., 2003, MNRAS 341, 669

Heber, U., 2009, ARA\&A 47, 211

Horner, J., Marshall, J. P., Wittenmyer, R. A., Tinney, C. G., 2011, MNRAS 416, L11

Jacobs, V. A., Ostensen, R. H., van Winckel, H., Bloeman, S., et al., 2011, AIPC 1331, 304

Kilkenny, D., 2011, MNRAS 412, 487

Kilkenny, D., Keuris, S., Marang, F., Roberts, G., van Wyk, F., Ogloza, W., 2000, The observatory 120,48

Kilkenny, D., O’Donoghue, D., Koen, C., Lynas-Gray, A. E., van Wyk, F., 1998, MNRAS 296,329

Kilkenny, D., Reed, M. D., O’Donoghue, D., Kawaler, S. D., et al., 2003, MNRAS 345, 834

Lee, J. W., Kim, S.-L., Kim, C.-H., Koch, R. H., Lee, C.-U., Kim, H.-I., Park, J.-H., 2009, AJ 137,3181 
Liao, W.-P. \& Qian, S.-B., 2010, MNRAS 405, 1930

Kraft, R. P., Matthews, J., Greenstein, J. L., 1962, ApJ 136, 312

Lee, J. W., Kim, S.-L., Kim, C.-H., Koch, R. H., Lee, C.-U., Kim, H.-I., Park, J.-H., 2009, AJ 137,3181

Menzies, J. W. \& Marang, F., 1986, Symposium of the International Astronomical Union 118,305

Ostensen, R. H., Green, E. M, Bloemen, S., Marsh, T. R., Laird, J. B., et al., 2010, MNRAS 408, L51

Ostensen, R. H., Oreiro, R., Drechsel, H., Heber, U., Baran, A., Pigulski, A., 2007, ASP Conf. Ser. 372, 483

Polubek, G., Pigulski, A., Baran, A., Udalski, A., 2007, ASP Conf. Ser. 372, 487

Pringle, J. E., 1975, MNRAS 170, 633

Qian, S.-B., Dai, Z.-B., Zhu, L.-Y., Liu, L., He, J.-J., Liao, W.-P., and Li, L.-J., 2008, ApJ $689, \mathrm{~L} 49$

Qian, S.-B., Liao, W.-P., Zhu, L.-Y., Dai, Z.-B., 2010, ApJ 708, L66

Qian, S.-B., Liu, L., Liao, W.-P., Li, L.-J., Zhu, L.-Y., et al., 2011, MNRAS 414, L16

Qian, S.-B., Zhu, L.-Y., Zola, S., Liao, W.-P., Liu, L., Li, L.-J., Winiarski, M., Kuligowska, E., Kreiner, J. M., 2009, ApJ 695, L163

Rappaport, S., Verbunt, F., Joss, P. C., 1983, ApJ 275, 713

Shimansky, V., Sakhibullin, N. A., Bikmaev, I., Ritter, H., Suleimanov, V., Borisov, N., Galeev, A., 2006, A\&A 456, 1069

Silvotti, R., Schuh, S., Janulis, R., Solheim, J.-E., Bernabei, S., Ostensen, R., Oswalt, T. D., Bruni, I., Gualandi, R., Bonanno, A., et al., 2007, Nature 449, 189

Spruit, H. C. \& Ritter, H., 1983, A\&A 124, 267

Stumpff, P., 1980, A\&AS 41, 1

Wils, P., Di Scala, G., Otero, S. A., 2007, Inf. Bull. Var. Stars No. 5800 
Table 1: New CCD times of light minimum of NY Vir.

\begin{tabular}{llllll}
\hline BJD (days) & Errors (days) & Min. & Filters & E & Telescopes \\
\hline \hline 2454211.16991 & 0.00007 & I & R & 39477 & The 1.0-m telescope \\
2455709.64037 & 0.00001 & I & I & 54311 & The 2.15-m telescope \\
2455709.69102 & 0.00004 & II & I & 54311.5 & The $2.15-\mathrm{m}$ telescope \\
2455711.55967 & 0.00001 & I & R & 54330 & The 2.15-m telescope \\
2455711.61016 & 0.00004 & II & R & 54330.5 & The $2.15-\mathrm{m}$ telescope \\
2455711.66070 & 0.00001 & I & V & 54331 & The $2.15-\mathrm{m}$ telescope \\
2455711.71125 & 0.00006 & II & V & 54331.5 & The 2.15-m telescope \\
2455712.56984 & 0.00002 & I & B & 54340 & The 2.15-m telescope \\
2455712.62042 & 0.00005 & II & B & 54340.5 & The 2.15-m telescope \\
\hline \hline
\end{tabular}

